\title{
Metabolic engineering of Moorella thermoacetica for thermophilic bioconversion of gaseous substrates to a volatile chemical
}

\author{
Junya Kato ${ }^{1 \dagger}$, Kaisei Takemura ${ }^{1 \dagger}$, Setsu Kato ${ }^{1}$, Tatsuya Fujii ${ }^{2}$, Keisuke Wada ${ }^{2}$, Yuki Iwasaki ${ }^{2}$, Yoshiteru Aoi ${ }^{1}$, \\ Akinori Matsushika ${ }^{1,2}$, Katsuji Murakami ${ }^{2}$ and Yutaka Nakashimada ${ }^{1 *}$ (1)
}

\begin{abstract}
Gas fermentation is one of the promising bioprocesses to convert $\mathrm{CO}_{2}$ or syngas to important chemicals. Thermophilic gas fermentation of volatile chemicals has the potential for the development of consolidated bioprocesses that can simultaneously separate products during fermentation. This study reports the production of acetone from $\mathrm{CO}_{2}$ and $\mathrm{H}_{2}, \mathrm{CO}$, or syngas by introducing the acetone production pathway using acetyl-coenzyme $\mathrm{A}$ (Ac-CoA) and acetate produced via the Wood-Ljungdahl pathway in Moorella thermoacetica. Reducing the carbon flux from Ac-CoA to acetate through genetic engineering successfully enhanced acetone productivity, which varied on the basis of the gas composition. The highest acetone productivity was obtained with $\mathrm{CO}-\mathrm{H}_{2}$, while autotrophic growth collapsed with $\mathrm{CO}_{2}-\mathrm{H}_{2}$. By adding $\mathrm{H}_{2}$ to $\mathrm{CO}$, the acetone productivity from the same amount of carbon source increased compared to CO gas only, and the maximum specific acetone production rate also increased from 0.04 to $0.09 \mathrm{~g}$-acetone/ $\mathrm{g}$-dry cell/h. Our development of the engineered thermophilic acetogen $M$. thermoacetica, which grows at a temperature higher than the boiling point of acetone $\left(58^{\circ} \mathrm{C}\right)$, would pave the way for developing a consolidated process with simplified and cost-effective recovery via condensation following gas fermentation.
\end{abstract}

Keywords: Gas fermentation, Metabolic engineering, Acetone production, Acetogen, Thermophile, Moorella thermoacetica

\section{Introduction}

Metabolic engineering of thermophilic microorganisms has several benefits compared to mesophilic microorganisms, such as a lower contamination risk, less energy required for cooling the fermentation system, and a faster production rate due to the advantageous thermodynamics (Sonnleitner and Fiechter 1983). One potential application is bioreactive distillation, which involves simultaneously fermenting volatile chemicals and collecting them by distillation (Zeldes et al. 2018). The

\footnotetext{
*Correspondence: nyutaka@hiroshima-u.ac.jp

† Junya Kato and Kaisei Takemura contributed equally to this work

1 Graduate School of Integrated Sciences for Life, Hiroshima University,

1-3-1 Kagamiyama, Higashihiroshima, Hiroshima 739-8530, Japan

Full list of author information is available at the end of the article
}

bioreactive distillation has fewer steps and a lower cost of purification of target chemicals compared to the conventional fermentation processes. In addition, maintenance of the low concentration of fermentation products prevents the exposure of the chemicals to bacteria that inhibits bacterial growth and metabolism.

Acetone is a volatile chemical used as an industrial solvent and a precursor of important downstream products (Anbarasan et al. 2012; Peters et al. 2015). Currently, industrial acetone production depends on petrochemical phenol production via the cumene process. Although the cumene process is cost-effective and widely used, it has the risk of shortage of nonrenewable fossil feedstock. Therefore, there is a demand for an alternative process, and acetone production by bioconversion from renewable feedstock is one option. 
Historically, acetone production by bioconversion has been studied as acetone-butanol-ethanol (ABE) fermentation. However, the focus is on alcohol production, and much effort is made for inhibiting acetone production as a by-product (Jiang et al. 2009; Luo et al. 2016; Xu et al. 2015). Metabolic engineering enables the development of acetone-producing strains such as Escherichia coli as the host (Bermejo et al. 1998). A few studies have reported thermophilic acetone fermentation from carbohydrates (Shaw et al. 2015; Straub et al. 2020). In addition, metabolic engineering of Synechocystis sp. PCC 6803, Acetobacterium woodii, and Clostridium ljungdahlii makes acetone production possible from $\mathrm{CO}_{2}$ or $\mathrm{CO}$ gas as the carbon source (Banerjee et al. 2014; Hoffmeister et al. 2016; Zhou et al. 2012) by mesophilic organisms. $\mathrm{CO}_{2}$ fixation by mixotrophy improves conversion of organic compounds to acetone, as shown by an engineered strain of C. ljungdahlii (Jones et al. 2016).

Among the various bioconversion applications, gas fermentation utilizing anaerobic acetogenic bacteria (acetogen) is attracting increasing attention. Gas fermentation is economically and environmentally friendly because inexpensive gaseous waste feedstock, such as steel mill waste gas or syngas primarily comprising $\mathrm{CO}$ and $\mathrm{H}_{2}$, can be used (Claassens et al. 2016; Durre and Eikmanns 2015; Liew et al. 2016). Whereas practical applications still demand higher productivity and cost-effective processes, the combination of bioreactive distillation as the purification process with gas fermentation can reduce waste and cost, in addition to engineering the metabolism of acetogens. The economic feasibility of acetone production from syngas by bioreactive distillation has been evaluated using hypothetical systems with engineered thermophilic strains of Moorella thermoacetica (Redl et al. 2017). The bioreactive distillation also has advantages to remove acetone that has inhibitory effect on the cell growth and could maintain high cell density without need to replace culture medium if appropriate bioreactors are used. However, the detailed metabolic design and construction of the thermophilic strains for gas fermentation, their availability, and, therefore, experimental data to support the system are missing.

This study genetically engineered the thermophilic homoacetogen $M$. thermoacetica to produce acetone from gaseous substrates at high temperature. We also developed a strategy to increase the carbon flux to acetone by genetic engineering and evaluated the productivity from $\mathrm{CO}_{2}-\mathrm{H}_{2}, \mathrm{CO}$ and $\mathrm{CO}-\mathrm{H}_{2}$ as a model syngas. To our knowledge, this is the first study to provide strains for thermophilic gas fermentation of acetone.

\section{Materials and methods}

Bacterial strains and growth conditions

We used M. thermoacetica ATCC 39073 and its derivatives in this study (Table 1). Modified ATCC1754 PETC medium comprising $1.0 \mathrm{~g}$ of $\mathrm{NH}_{4} \mathrm{Cl}, 0.1 \mathrm{~g}$ of $\mathrm{KCl}, 0.2 \mathrm{~g}$ of

Table 1 Strains and plasmids used in this study

\begin{tabular}{|c|c|c|}
\hline Strain or plasmid & Description & $\begin{array}{l}\text { Source or } \\
\text { reference }\end{array}$ \\
\hline \multicolumn{3}{|l|}{ Strains } \\
\hline \multicolumn{3}{|l|}{ Escherichia coli } \\
\hline HST08 & Cloning host & TaKaRa \\
\hline TOP10 & Modification host & Invitrogen \\
\hline \multicolumn{3}{|l|}{ M. thermoacetica } \\
\hline ATCC 39073 & Wild-type strain & ATCC \\
\hline$\Delta p y r F$ & pyrF gene was deleted & Kita et al. (2013) \\
\hline pyrF::acetone & The thermophilic acetone operon was introduced into the pyrF region by using pHM17 & This study \\
\hline pduL2::acetone & $\begin{array}{l}\text { The thermophilic acetone operon was introduced into the pduL2 region by using pHM5. As a result, pduL2 } \\
\text { was knocked out }\end{array}$ & This study \\
\hline \multicolumn{3}{|l|}{ Plasmids } \\
\hline pBAD33 & Backbone plasmid for methylation plasmids, $\mathrm{Cm}^{\mathrm{r}}$ & Guzman et al. (1995) \\
\hline pBAD-M1281 & pBAD33 carrying the Moth_1671, Moth_1672, and Moth_2281 for DNA methylation & Kita et al. (2013) \\
\hline pK18mob & Backbone plasmid for transformation plasmids, $\mathrm{Km}^{\mathrm{r}}$ & Schäfer et al. (1994) \\
\hline pK18-Idh & A transformation plasmid to introduce $I d h$ into the $p y r F$ region by using $p y r F$ as a selection marker & Kita et al. (2013) \\
\hline pK18- $\Delta p d u L 2:: / d h$ & A transformation plasmid to introduce $I d h$ into the $p d u L 2$ region by using $p y r F$ as a selection marker & Iwasaki et al. (2017) \\
\hline pHM17 & $\begin{array}{l}\text { A transformation plasmid to introduce the thermophilic acetone operon into the } p y r F \text { region by using pyrF } \\
\text { as a selection marker }\end{array}$ & This study \\
\hline pHM5 & $\begin{array}{l}\text { A transformation plasmid to introduce the thermophilic acetone operon into the pduL2 region by using pyrF } \\
\text { as a selection marker }\end{array}$ & This study \\
\hline
\end{tabular}


$\mathrm{MgSO}_{4} \cdot 7 \mathrm{H}_{2} \mathrm{O}, 0.8 \mathrm{~g}$ of $\mathrm{NaCl}, 0.1 \mathrm{~g}$ of $\mathrm{KH}_{2} \mathrm{PO}_{4}, 0.02 \mathrm{~g}$ of $\mathrm{CaCl}_{2} \cdot 2 \mathrm{H}_{2} \mathrm{O}, 2.0 \mathrm{~g}$ of $\mathrm{NaHCO}_{3}, 10 \mathrm{~mL}$ of trace elements, $10 \mathrm{~mL}$ of Wolfe's vitamin solution (Tanner 1989), and $1.0 \mathrm{mg}$ of resazurin/L of deionized water was used as the basal medium (Tanner et al. 1993). The $\mathrm{pH}$ was adjusted to 6.9. The medium was prepared anaerobically by boiling and cooling under a $\mathrm{N}_{2}-\mathrm{CO}_{2}$ (80:20) mixed-gas atmosphere. After cooling, the medium was dispensed to $125-\mathrm{mL}$ serum bottles under a $\mathrm{N}_{2}-\mathrm{CO}_{2}$ mixed-gas atmosphere. The serum bottles were crimp-sealed and autoclaved.

Before starting culture, we added yeast extract and L-cysteine $\cdot \mathrm{HCl} \cdot \mathrm{H}_{2} \mathrm{O}$ to reach a final concentration of 1.0 and $1.2 \mathrm{~g} / \mathrm{L}$, respectively. $2.0 \mathrm{~g} / \mathrm{L}$ of fructose was added for routine cultivation and to examine acetone production from sugar. The final volume was adjusted to $50 \mathrm{~mL}$. To add gas substrates, we replaced the headspace of the serum bottles by $\mathrm{CO}_{2}-\mathrm{H}_{2}(20: 80)(0.1 \mathrm{MPa})$, or we added $\mathrm{CO}(0.04 \mathrm{MPa})$ and additional $\mathrm{H}_{2}(0.04 \mathrm{MPa})$ after replacing the headspace of the serum bottles with $\mathrm{N}_{2}$ gas at atmospheric pressure. The temperature was maintained at $55^{\circ} \mathrm{C}$ with shaking at $180 \mathrm{rpm}$.

\section{Plasmid construction}

We constructed two plasmids, pHM17 and pHM5, to introduce the thermophilic acetone operon into the pyrF or the $p d u L 2$ region of the chromosome in M. thermoacetica (Table 1). We synthesized the thermophilic acetone operon under the constitutive glyceraldehyde3-phosphate dehydrogenase (G3PD) promoter after codon optimization of the four genes encoding acetone biosynthetic enzymes for expression in $M$. thermoacetica (GenScript). The genes constituting the thermophilic acetone operon were $c t f A$ (Tmel_1136) and ctfB (Tmel_1135) from Thermosipho melanesiensis, thl (TTE0549) from Caldanaerobacter subterraneus subsp. tengcongensis, and adc (CA_P0165) from C. acetobutylicum. The open reading frames coding these four genes were driven by the constitutive G3PD promoter (Kita et al. 2013), and the gene order was determined on the basis of the biochemical information about the enzymes: activity, stability, and complex formation (Zeldes et al. 2018). Each gene was separated by an intergeneic spacer with a ribosome-binding site, and the DNA fragment synthesized was amplified by polymerase chain reaction (PCR) using KOD plus ver.2 (Toyobo Co., Ltd., Osaka, Japan) and this synthetic operon was inserted into the plasmids with a $p y r F$ marker in either the $p y r F$ or the $p d u L 2$ region using the In-Fusion HD cloning kit (Clontech Laboratories, TaKaRa Bio, Shiga, Japan). We used pK18-ldh (Kita et al. 2013) or pK18- $\Delta p d u L 2:: l d h$ (Iwasaki et al. 2017) as a template to amplify the plasmids (Table 1). Table 2 lists the primers used for PCR. We used JK50 and JK51 to amplify
Table 2 PCR primers used in this study

\begin{tabular}{ll}
\hline Name & Sequence $\left(\mathbf{5}^{\prime}\right.$ to $\mathbf{3}^{\prime}$ ) \\
\hline JK50 & GGTGAAATAATAACTGGACGGTTGCCAAGTACCG \\
JK51 & ATGAAAGCAGGCCGATTACTTCAGATAATCGTAGATCAC \\
& TTCGG \\
$J K 52$ & TCGGCCTGCTTTCATGCTTG \\
$J K 53$ & AGTTATTATTTCACCATCTCTATTTCCGCC \\
JK226 & GGCCGCCGCCATTTAGCATATCAAGAG \\
JK227 & GCCGCAAATGCTGGTAAAGGCTATC \\
$1181-$ up-F & CGTTCAATAGGAAGACCACAG \\
$1181-d w-R$ & GCAGTAAGCTGTATCGCAATG \\
\hline
\end{tabular}

the insert and JK52 and JK53 to amplify plasmid backbones. Finally, we cloned the constructed plasmids in E. coli HST08 and confirmed the DNA sequences using Sanger sequencing.

\section{Transformation and selection of mutants}

We performed the genetic transformation of $M$. thermoacetica, as previously described (Kita et al. 2013). All procedures were performed under aerobic conditions, except for cell growth. Briefly, we cultured the $M$. thermoacetica $\triangle$ pyrF mutant to the mid-log phase in basal medium supplemented with $2 \mathrm{~g} / \mathrm{L}$ of fructose as a carbon source and $10 \mu \mathrm{g} / \mathrm{mL}$ of uracil instead of yeast extract, and harvested it by centrifugation. Next, the cells were washed twice with $272 \mathrm{mM}$ sucrose solution and used for electroporation with methylated DNA in the E. coli TOP10-harboring plasmid pBAD-M1281. The transformed cells were then cultured at $55^{\circ} \mathrm{C}$ for $24-48 \mathrm{~h}$ with a low uracil concentration of $1 \mu \mathrm{g} / \mathrm{mL}$ before inoculation to the modified ATCC1754 PETC medium containing agar without uracil or yeast extract in roll tubes (Hungate 1969). The roll tubes were cultured at $55{ }^{\circ} \mathrm{C}$, and the colonies were subcultured to confirm the insertion of the thermophilic acetone operon by using PCR. We used the primer set JK226 and JK227 to amplify the pyrF region and 1181-up-F and 1181-up-R to amplify the pduL2 region. The constructed strain with higher acetone productivity has been deposited to NITE (NITE AP-03217).

\section{Analytical methods}

We sampled and analyzed $1 \mathrm{~mL}$ of the culture medium at each time point and calculated the dry cell weight using the optical density (OD) at $600 \mathrm{~nm}(1 \mathrm{~g}$ [dry cell weight] $/ \mathrm{L}=0.383$ OD) (Iwasaki et al. 2017). The culture supernatant was analyzed for the amount of fructose, formate, acetate, and acetone using high-performance liquid chromatography (HPLC) (LC-2000 Plus HPLC; Jasco, Tokyo, Japan) equipped with a refractive index detector (RI-2031 Plus; Jasco), a Shodex RSpak KC-811 column 


\begin{abstract}
(See figure on next page.)
Fig. 1 Design and construction of acetone-producing Moorella thermoacetica strains. a Acetone production pathway. Two molecules of Ac-CoA are converted to one molecule of acetone via three reactions using one molecule of acetate. The reactions release a CoA molecule, an Ac-CoA molecule, and a $\mathrm{CO}_{2}$ molecule, in addition to an acetone molecule. Acetate pathway from Ac-CoA is also shown. Ac-CoA is converted to acetyl phosphate by phosphotransacetylase that is encoded by pduL 1 as well as pduL2, followed by conversion to acetate. $\mathbf{b}$ Schematic representation of the synthetic acetone production operon. Genes and promoters are shown by block and fine arrows, respectively. c, e Schematic representations of the introduction of the synthetic thermophilic acetone operon by homologous recombination into the pyrF (c) and the pduL2 (e) region. The gray boxes highlight DNA regions used for recombination, and the line arrows represent primers used for PCR. The primer set to amplify the pyrF region is JK226 and JK227 and that for the pduL2 region is 1181-up-F and 1181-dw-R. d, f Verification of the presence of the thermophilic acetone operon in the $\operatorname{pyr} F(\mathbf{d})$ and the $\operatorname{pduL2}(\mathbf{f})$ region. The genomic region of each gene was amplified by PCR, and the size shift due to the insertion was confirmed. The size of the PCR product of the pyrF region shifted from 0.5 to $4.8 \mathrm{~kb}$ by introducing the thermophilic acetone operon and the selection marker (d). Similarly, the PCR product of the pduL2 region shifted from 1.0 to $4.9 \mathrm{~kb}(\mathbf{f})$
\end{abstract}

(Showa Denko, Kanagawa, Japan), and a Shodex RSpak KC-G guard column (Showa Denko) at $60{ }^{\circ} \mathrm{C}$. Ultrapure water containing $0.1 \%(\mathrm{v} / \mathrm{v})$ phosphoric acid was used as the mobile phase at a flow rate of $0.7 \mathrm{~mL} / \mathrm{min}$, and crotonate was used as an internal standard (Miura et al. 2014). The gas composition in the headspace of the serum bottles was analyzed by using GC-8A gas chromatography (Shimadzu, Kyoto, Japan) equipped with a thermal conductivity detector and a stainless steel column packed with activated carbon at $70{ }^{\circ} \mathrm{C}$. Argon was used as the carrier gas (Miura et al. 2014). The amount of dissolved carbonate in the culture medium was measured by using a total organic carbon analyzer (TOC-L; Shimadzu).

\section{Nucleic acid sequences}

The nucleic acid sequences of the synthetic acetone operon have been deposited to GenBank (accession number MW436696).

\section{Results}

Design and construction of genetically engineered $M$. thermoacetica strains for thermophilic acetone production Moorella thermoacetica grows at $45{ }^{\circ} \mathrm{C}-65{ }^{\circ} \mathrm{C}$. A pathway for thermophilic acetone production, which functions up to $70{ }^{\circ} \mathrm{C}$, has been proposed with enzyme candidates (Zeldes et al. 2018). This pathway converted two molecules of acetyl-CoA (Ac-CoA) to acetoacetylCoA (Acac-CoA) as the start reaction by thiolase (Thl), followed by two reactions that produced acetoacetate (Acac) and acetone (Fig. 1a). When Acac was produced by CoA transferase (CtfAB), one molecule of acetate was required to receive a $\mathrm{CoA}$ molecule from Acac-CoA. $M$. thermoacetica provides both Ac-CoA and acetate that are used as substrates in this pathway on sugars or gaseous substrates. In M. thermoacetica, Ac-CoA is an intermediate to produce acetate as the end metabolite. We selected thermophilic enzymes and designed the acetone biosynthesis operon (Fig. 1b), and the synthetic thermophilic acetone operon was successfully introduced into the wild-type (WT) background of M. thermoacetica (Fig. 1c and d). Next, we cultured the pyrF::acetone strain in basal medium supplemented with fructose at $55{ }^{\circ} \mathrm{C}$ (optimum growth temperature). Acetone was successfully produced and released into the culture supernatant, indicating functional expression of the enzymes. Consistent with the absence of homologous genes encoding secondary alcohol dehydrogenase in the genome of $M$. thermoacet$i c a$, the produced acetone was not converted to isopropanol unlike the case of some acetogens (Hoffmeister et al. 2016; Kopke et al. 2014; Pierce et al. 2008). However, we detected a large amount of acetate (about three times more than acetone) in the culture supernatant, indicating that Ac-CoA is mostly converted to acetate (Fig. 2a, b and e).

\section{Deletion of pduL2 and preservation of pduL1 lead to higher acetone production}

The introduction of the thermophilic acetone operon did not cause high acetone production by $M$. thermoacetica. We hypothesized that the Thl responsible for the first reaction could not capture Ac-CoA because of the abundant phosphotransacetylase activity by PduL1 and PduL2 in $M$. thermoacetica. PduL2 showed more than a tenfold lower Michaelis constant $\left(K_{\mathrm{m}}=0.04 \mathrm{mM}\right)$ against Ac-CoA compared to PduL1 $\left(K_{\mathrm{m}}=0.49 \mathrm{mM}\right)$, while Thl showed a $K_{\mathrm{m}}$ value of $0.27 \mathrm{mM}$ (Breitkopf et al. 2016; Loder et al. 2015). Although we did not measure PduL1, PduL2, Thl, and Ac-CoA levels in the cells, the low $K_{\mathrm{m}}$ value of PduL2 might explain the abundant acetate production in the pyrF::acetone strain. To test this hypothesis, we knocked out $p d u L 2$ and measured the acetone production. We introduced the thermophilic acetone operon to replace the $p d u L 2$ coding region, which enabled us to delete $p d u L 2$ and introduce acetone biosynthetic genes at the same time (Fig. 1e and $\mathrm{f}$ ). We cultured the pduL2::acetone strain in basal medium with fructose and found a significant increase in acetone production and decrease in acetate production, resulting in $1.0 \pm 0.02 \mathrm{~mol}$-acetone $/ \mathrm{mol}$-fructose and $0.45 \pm 0.03 \mathrm{~mol}$-acetate $/ \mathrm{mol}$-fructose (Fig. $2 \mathrm{c}-\mathrm{e}$ ). The 
a

\section{Acetone pathway}
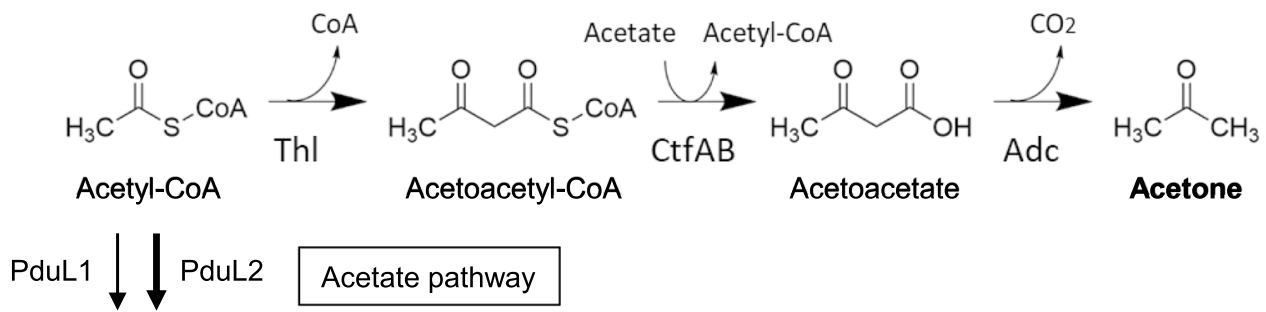

Acetyl phosphate $\longrightarrow$ Acetate

b

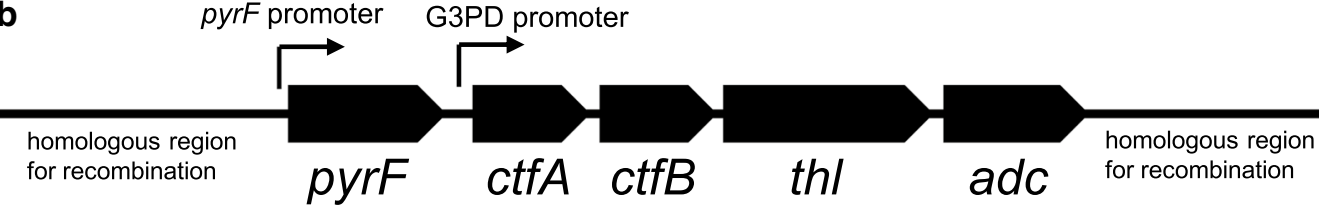

C
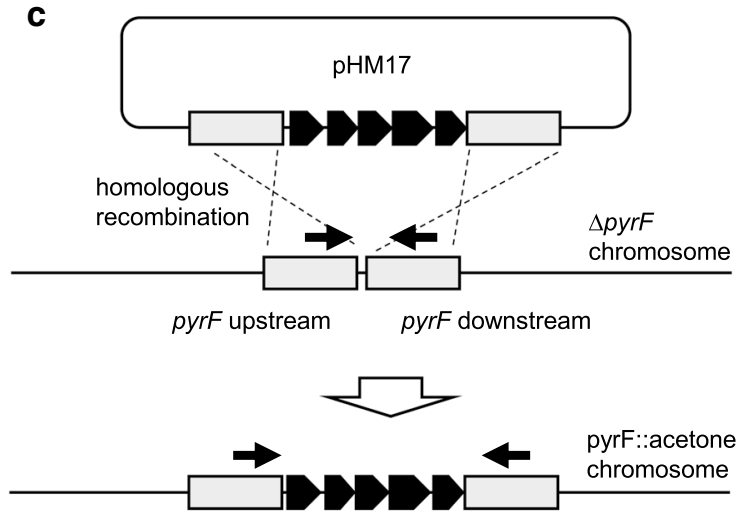

-
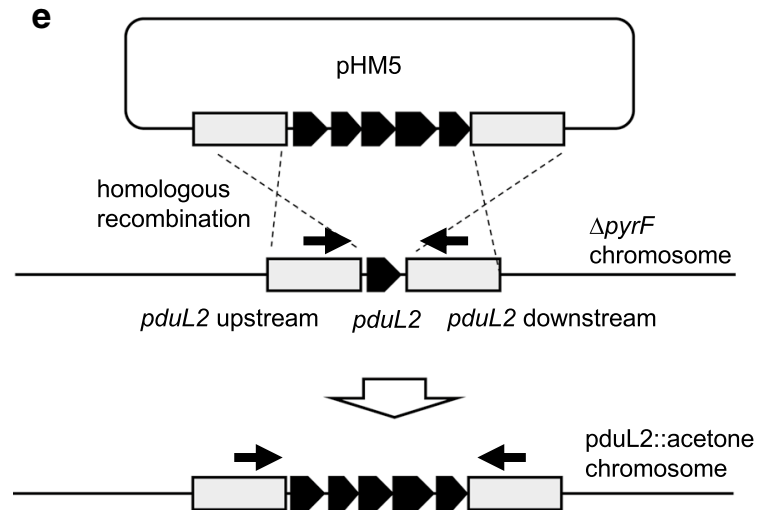

d

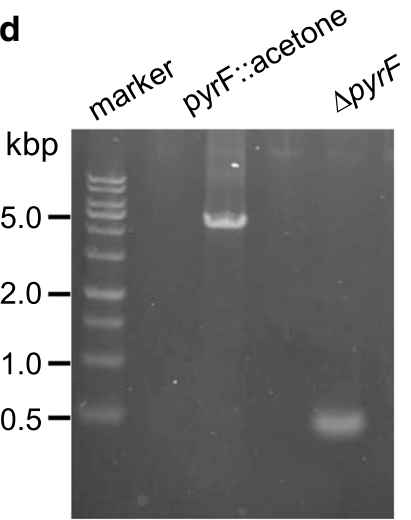

$\mathbf{f}$

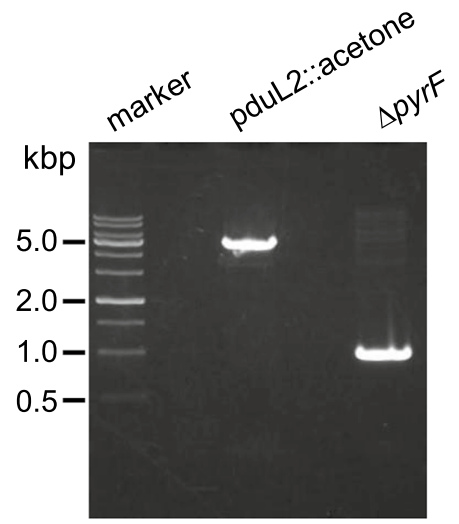

acetone-acetate ratio was $0.35 \pm 0.03$ in the case of the pyrF::acetone strain, but increased to $2.23 \pm 0.21$ in the pduL2::acetone strain. Acetone production was dominant over acetate production, and thus, we successfully directed more Ac-CoA pool to the acetone pathway.
Thermophilic acetone production from $\mathrm{CO}_{2}-\mathrm{H}_{2}$

We aimed to produce acetone from gaseous substrates with high productivity by using the pduL2::acetone strain. $\mathrm{CO}_{2}$ and $\mathrm{H}_{2}$ are the best-studied form of substrates for autotrophic acetogenesis. First, we tested $\mathrm{CO}_{2}$ 

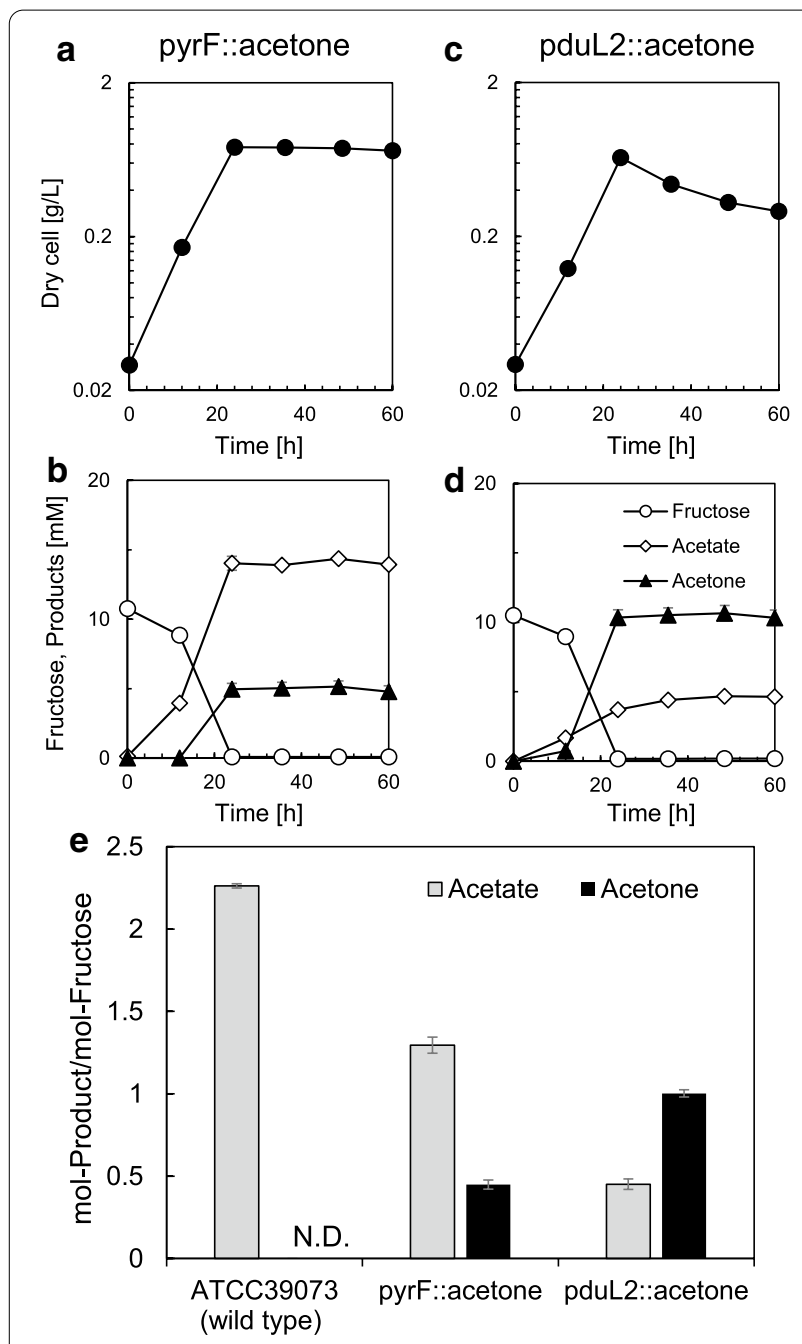

Fig. 2 Acetone and acetate production from fructose by the recombinant Moorella thermoacetica strains. a, c Dry cell weight according to the OD for the pyrF::acetone strain (a) and pduL2::acetone strain (c). b, d Concentration of fructose and excreted metabolites in the culture supernatant measured by HPLC for the pyrF::acetone strain (b) and the pduL2::acetone strain $(\mathbf{d})$. Data represent the mean with SDs of three biological replicates. Most error bars are smaller than the symbols of data plots. $\mathbf{e}$ The acetone and acetate productivity per 1 mol of fructose is shown with black (acetone) and gray (acetate) bars. The productivity was calculated based on the measurement after complete consumption of the supplemented fructose. The parental strain, ATCC 39073 (wild type), which does not produce acetone is shown for comparison. Data represent the mean with SDs of three biological replicates

as a carbon source and $\mathrm{H}_{2}$ as an energy source. To set up the culture, the bacterial strain was grown in basal medium supplemented with fructose, and we used this culture to inoculate fresh medium with $\mathrm{CO}_{2}-\mathrm{H}_{2}$ in the headspace of the vial for the pre-culture. This step was performed for bacterial cells to completely consume fructose, followed by adaptation to $\mathrm{CO}_{2}-\mathrm{H}_{2}$ metabolism. We
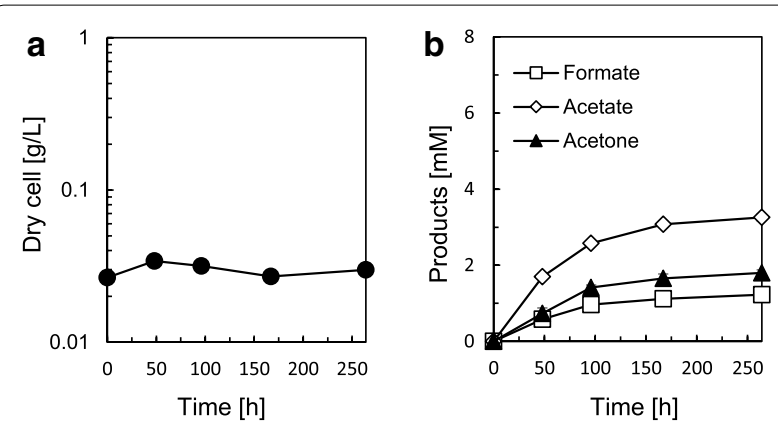

Fig. 3 Growth and metabolite profile of the pduL2::acetone strain in $\mathrm{CO}_{2}-\mathrm{H}_{2}$ as the substrate. a Dry cell weight according to the OD. $\mathbf{b}$

Concentration of the excreted metabolites in the culture supernatant measured by HPLC. Data represent the mean with SDs of three biological replicates. Most error bars are smaller than the symbols of data plots

inoculated fresh medium supplemented with $\mathrm{CO}_{2}-\mathrm{H}_{2}$ by using the adapted cells and recorded the culture profile. There was almost no growth during $254 \mathrm{~h}$ of cultivation time (Fig. 3a). Excreted metabolites accumulated over time (Fig. 3b), indicating that the cells were metabolically active. Acetone was successfully produced in $\mathrm{CO}_{2}-\mathrm{H}_{2}$, reaching $1.8 \pm 0.08 \mathrm{mM}$ in the culture supernatant after $254 \mathrm{~h}$. Acetate production reached $3.3 \pm 0.09 \mathrm{mM}$, which was dominant over acetone production, although the pduL2::acetone strain was engineered to have a higher carbon flux to acetone in the culture supplemented with fructose. In addition, formate, which is an intermediate in the Wood-Ljungdahl pathway (WLP), also accumulated in the culture supernatant, reaching $1.2 \pm 0.12 \mathrm{mM}$, indicating that the metabolic flow of the WLP is affected.

\section{Thermophilic acetone production from $\mathrm{CO}$ or syngas}

The pduL2::acetone strain showed no growth in $\mathrm{CO}_{2}-$ $\mathrm{H}_{2}$, so we tested a more energetically favorable gas, $\mathrm{CO}$, for acetone production with autotrophic growth. $M$. thermoacetica uses $\mathrm{CO}$ as the energy source and shows a higher biomass than in $\mathrm{H}_{2}$ because of higher adenosine triphosphate (ATP) generation ( $\mathrm{Hu}$ et al. 2016; Kerby and Zeikus 1983). To initiate the culture, we adapted the pduL2::acetone strain to $\mathrm{CO}$ in the same way as $\mathrm{CO}_{2}-\mathrm{H}_{2}$, especially because $\mathrm{CO}$ inhibits $M$. thermoacetica growth without adaptation (Kerby and Zeikus 1983). The bacterial cells proliferated using CO as a carbon and energy source, in contrast to $\mathrm{CO}_{2}-\mathrm{H}_{2}$ as observed by an obvious increase of the cellular biomass (Fig. 4a). We also observed acetone and acetate production, and their maximum concentration was $1.1 \pm 0.04$ and $4.2 \pm 0.08 \mathrm{mM}$, respectively (Fig. $4 \mathrm{~b}$ ). The acetone-acetate ratio was $0.27 \pm 0.01$, which was again acetate dominant. No formate production was observed 

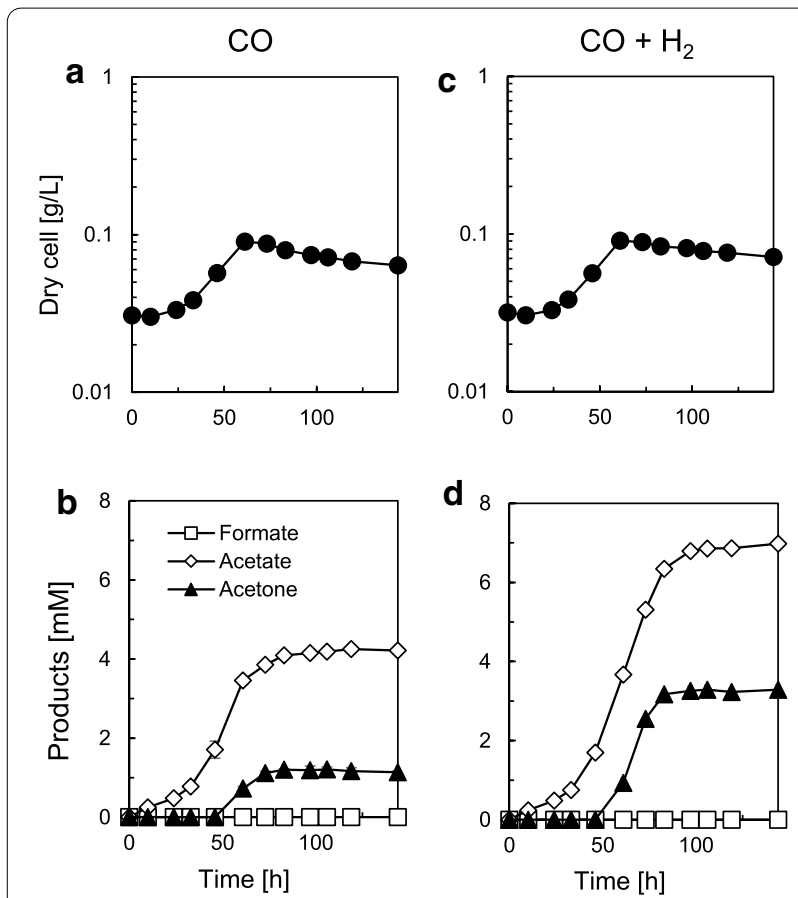

Fig. 4 Growth and metabolite profile of the pduL2::acetone strain in $\mathrm{CO}$ and $\mathrm{CO}-\mathrm{H}_{2}$. a, c Dry cell weight according to the OD. $\mathbf{b}, \mathbf{d}$ Excreted metabolites measured by HPLC. Profiles of $\mathrm{CO}(\mathbf{a}, \mathbf{b})$ and $\mathrm{CO}-\mathrm{H}_{2}(\mathbf{c}, \mathbf{d})$ are shown, respectively. The values shown are means of three biological replicates, and error bars represent the SD. Some error bars showing a small error range overlapped with symbols of data plots

in contrast to $\mathrm{CO}_{2}-\mathrm{H}_{2}$, indicating that the metabolic flow of WLP was not affected. Thus, the pduL2::acetone strain autotrophically grew and produced acetone in CO.

Syngas mainly comprises $\mathrm{CO}$ and $\mathrm{H}_{2}$ and applicable substrates for sustainable gas fermentation. We tested a 1:1 $\mathrm{CO}$ and $\mathrm{H}_{2}$ mixture as a model case. The culture was set up in the same way as in CO. Whereas the culture profile showed almost the same biomass and growth rate as in $\mathrm{CO}$ without $\mathrm{H}_{2}$ (Fig. 4c), the acetone productivity significantly improved from $1.1 \pm 0.04$ to $3.3 \pm 0.10 \mathrm{mM}$ (Fig. $4 \mathrm{~b}$ and d) from the same amount of $\mathrm{CO}$, and the increment was higher compared to acetate (from $4.2 \pm 0.08$ to only $7.0 \pm 0.10 \mathrm{mM}$ ), indicating enhanced carbon flux to the acetone production pathway. The acetone-acetate ratio increased from $0.27 \pm 0.01$ to $0.47 \pm 0.01$ and was still acetate dominant but was higher compared to with CO. In addition, there was no formate accumulation. Therefore, by adding $\mathrm{H}_{2}$, the acetone productivity from the same amount of carbon source increased, and the maximum specific acetone production rate also increased from $0.04 \pm 0.003$ to $0.09 \pm 0.005$ g-acetone $/$ g-dry cell/h.
The growth and metabolite profiles of the autotrophic acetone production in $\mathrm{CO}$-containing gases can be compared by the fermentation parameters summarized in Table 3. The electrons derived from $\mathrm{H}_{2}$ seemed invested to acetone and acetate production rather than cellular biomass, because $\mathrm{H}_{2}$ supplementation did not affect cell growth. It was also indicated that the electron input was directed to acetone rather than acetate.

\section{Discussion}

Acetone production by introduction of a thermophilic acetone biosynthetic operon in $M$. thermoacetica showed that the selected proteins were functionally expressed. However, when WT background was used as the host, the end product was acetate dominant. To increase the acetone productivity over acetate, we made use of a unique feature of $M$. thermoacetica that two functional phosphotransacetylase genes ( $p d u L 1$ and $p d u L 2)$ are involved in acetate production. In the pyrF::acetone strain, three enzymes, PduL1 and PduL2 for acetate production and Thl for acetone production, compete to process Ac-CoA (Fig. 1a). PduL2 shows a lower $K_{\mathrm{m}}$ against Ac-CoA compared to PduL1 and Thl, and was likely to cause dominant acetate production. The removal of $p d u L 2$ successfully enhanced acetone production in the pduL2::acetone strain. As a result, the acetone-acetate ratio significantly increased to be comparable to the engineered C. ljungdahlii using lactose-inducible promoter for expression of the acetone synthesis enzymes under fructose or $\mathrm{CO}$ fermentation growth conditions (Banerjee et al. 2014). The acetone production ratio of our strain from $\mathrm{CO}$ was further increased by adding $\mathrm{H}_{2}$ as discussed below. Previously, the effect of $p d u L 2$ knockout was also seen in our report that partial disruption of the acetate production pathway by $p d u L 2$ knockout enhanced lactate production in the metabolically engineered strains to produce lactate (Iwasaki et al. 2017). The production of lactate, which is provided by a reduction of pyruvate, was significantly enhanced by eliminating $p d u L 2$ because of the increased available Ac-CoA pool and therefore pyruvate, while $p d u L 1$ disruption had a marginal effect.

It is also useful to control the metabolic flow toward acetate by eliminating only $p d u L 2$ to maintain the autotrophy on syngas. Acetate not only acts as a substrate for acetone synthesis but also sustains sufficient net ATP production by substrate-level phosphorylation. The pduL2::acetone strain maintains autotrophy in COcontaining gases, while autotrophic growth collapses in $\mathrm{CO}_{2}-\mathrm{H}_{2}$.

The autotrophy of acetogens is energetically at the limit of thermodynamics (Schuchmann and Muller 2014). When $M$. thermoacetica grows in $\mathrm{CO}_{2}-\mathrm{H}_{2}$, the net ATP production would be calculated to be only $0.5 \mathrm{~mol}-\mathrm{ATP} /$ 


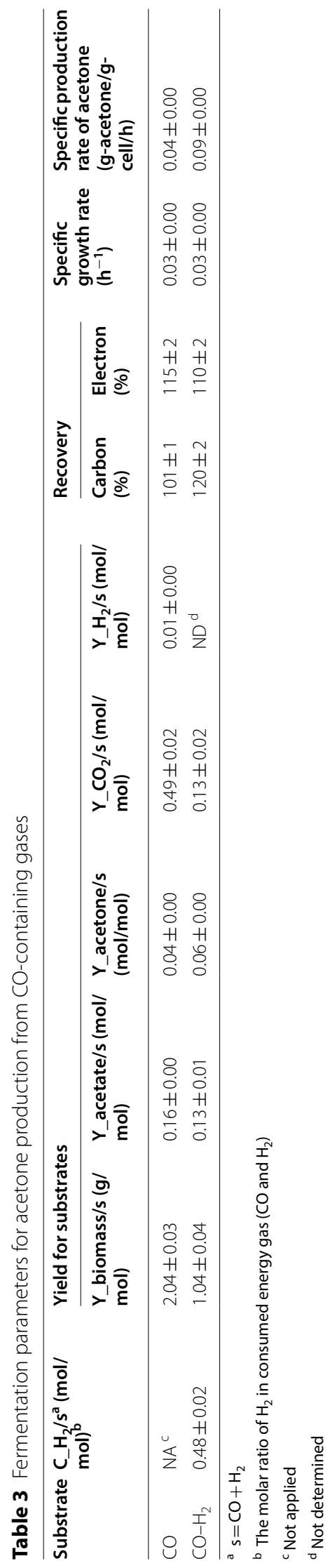


mol-acetate (Schuchmann and Muller 2014, and see also Online Resource Additional file 1: Figure S1):

$$
2 \mathrm{CO}_{2}+4 \mathrm{H}_{2} \rightarrow \text { Acetate }+2 \mathrm{H}_{2} \mathrm{O}(+0.5 \text { ATP })
$$

The positive ATP level is possible only when acetate is produced, because the ATP level is -0.5 at the point of Ac-CoA production ( $-0.5 \mathrm{~mol}-\mathrm{ATP} / \mathrm{mol}-\mathrm{Ac}-\mathrm{CoA})$. The consumed ATP is complemented by acetate production, which yields $1 \mathrm{~mol}-\mathrm{ATP} / \mathrm{mol}$-acetate. Because acetone production uses both Ac-CoA and acetate as substrates, diverging Ac-CoA to the acetone pathway lowers ATP production derived from substrate-level phosphorylation. When acetone is produced at the maximum efficiency from $\mathrm{H}_{2}$ and $\mathrm{CO}_{2}$, incorporating all the produced acetate, the net ATP is zero (Additional file 1: Fig. S1):

$$
3 \mathrm{CO}_{2}+8 \mathrm{H}_{2} \rightarrow \text { Acetone }+5 \mathrm{H}_{2} \mathrm{O}(+0 \mathrm{ATP})
$$

The pduL2::acetone strain did not grow in $\mathrm{CO}_{2}-\mathrm{H}_{2}$ (Fig. 3a), which can be explained by the low net ATP production. In fact, formate accumulation was observed in the metabolite analysis (Fig. 3b), indicating ATP shortage. In the WLP, formate is produced by the reduced nicotinamide adenine dinucleotide phosphate (NADPH)-dependent reduction of $\mathrm{CO}_{2}$ in $M$. thermoacetica. This formate is then ligated to tetrahydrofolate (THF) via an ATPdependent reaction (Schuchmann and Muller 2014). Therefore, low net ATP production causes ATP shortage and formate accumulation. In addition to formate, acetone and acetate are produced by the pduL2::acetone strain, indicating that the cells were metabolically active but not able to grow. Acetate production is linked to ATP production by substrate-level phosphorylation, and the ATP produced is used for cellular maintenance and formate ligation to THF. In the case of the engineered A. woodii producing acetone from $\mathrm{CO}_{2}-\mathrm{H}_{2}$, highly abundant acetate compared to acetone was produced to provide sufficient ATP production and maintain its autotrophic growth (Hoffmeister et al. 2016). ATP shortage is a challenge for the autotrophic acetone production with low level of acetate as the byproduct in $\mathrm{CO}_{2}-\mathrm{H}_{2}$.

In contrast, when a CO-containing gas is used, acetone production occurs as follows (Additional file 1: Fig. S1):

$$
8 \mathrm{CO}+3 \mathrm{H}_{2} \mathrm{O} \rightarrow \text { Acetone }+5 \mathrm{CO}_{2}(+2 \mathrm{ATP})
$$

In addition, when $\mathrm{H}_{2}$ is supplied (Additional file 1: Fig. S1),

$$
3 \mathrm{CO}+5 \mathrm{H}_{2} \rightarrow \text { Acetone }+2 \mathrm{H}_{2} \mathrm{O}(+0.75 \mathrm{ATP}) .
$$

In both cases, the net ATP is positive to sustain autotrophic growth. It has been discussed that when acetate is not formed from Ac-CoA to divert metabolic pathway, the WLP would be severely ATP limited
(Fast and Papoutsakis 2012). However, the acetone pathway utilizes acetate that is formed from Ac-CoA (Fig. 1a), which is advantageous to supply ATP. Applying $\mathrm{H}_{2}$ enhances the acetone production per consumed $\mathrm{CO}$ from $0.13 \mathrm{~mol}$-acetone $/ \mathrm{mol}-\mathrm{CO}$ without $\mathrm{H}_{2}$ to 0.33 mol-acetone/mol-CO with $\mathrm{H}_{2}$. In theory, acetone production should be 2.5 times higher with $\mathrm{H}_{2}$ supplementation, leaving no acetate as a by-product, when the reaction proceeds at the maximum efficiency. Our experiment with the pduL2::acetone strain showed that $\mathrm{H}_{2}$ supplementation significantly improved acetone production to $\sim 2.5$ times higher (Fig. 4) compared to only CO supplementation, although the amount of acetate also increased to $\sim 1.7$ times higher. The remaining acetate not incorporated into the acetone pathway indicates that acetone productivity could be potentially improved in both $\mathrm{CO}$ and $\mathrm{CO}-\mathrm{H}_{2}$ by tuning the final amount of acetate to zero without losing autotrophic growth.

One explanation for the abundant acetate that remained in our gas fermentation is due to the limit of enzymatic reactions, such as the CoA transferase that transfers CoA from Acac-CoA to acetate. An increase in acetate concentration is required to start solventogenesis in $C$. acetobutylicum, because $\mathrm{CoA}$ transferase shows a high $K_{\mathrm{m}}$ of $1200 \mathrm{mM}$ against acetate, while it shows a low $K_{\mathrm{m}}$ of $\sim 7-56 \mu \mathrm{M}$ against Acac-CoA (Wiesenborn et al. 1989). Although we did not analyze the enzymatic properties of CoA transferase from $T$. melanesiensis, it is conceivable that the enzyme has a high $K_{\mathrm{m}}$ against acetate and that the acetate concentration is a limiting factor. In fact, culture on fructose provided much higher concentration of acetate (Fig. 2d). Further examination and optimization of the selected enzymes would contribute to higher productivity, in addition to the experiments such as utilization of bioreactors to provide abundant substrates to reach high concentrations of the products including acetate. It is also possible that PduL1, which is responsible for remained production of acetate in the pduL2::acetone strain, was expressed higher on the gaseous substrates. This is because when the acetone production rate was compared between fructose culture and $\mathrm{CO}-\mathrm{H}_{2}$ culture, both showed similar rates $(0.12 \pm 0.01 \mathrm{~g}$-acetone/g-dry cell/h on fructose and $0.09 \pm 0.00$ g-acetone/g-dry cell/h on $\mathrm{CO}-\mathrm{H}_{2}$, respectively, calculated from Figs. $2 \mathrm{~d}$ and $4 \mathrm{~d}$ ). In other words, ATP would not be limiting factor in $\mathrm{CO}-\mathrm{H}_{2}$ culture, owing to the sufficient production of acetate linked to ATP production. This level of acetate might be necessary for the autotrophic acetone production at this rate. Otherwise, repression of PduL1 expression or replacement of the enzyme itself with its homologue with larger $K_{\mathrm{m}}$ value 
would be able to reduce acetate production and increase acetone productivity.

Finally, yet importantly, acetone production by engineering acetogenic metabolism has the benefit of redox balance, in addition to the use of Ac-CoA and acetate as substrates. In many cases of redox balance by native and engineered metabolism, unused electrons in the metabolic pathways are dedicated (or disposed of) to the reactions for end products. The redox imbalance is often a cause of low yield of end products and poor bacterial growth. The acetone pathway from Ac-CoA requires no reducing energy, so the redox balance in acetone production is difficult by using, for example, an E. coli system under anaerobic conditions because of the absence of reactions for unused electrons (Bermejo et al. 1998). However, the WLP produces acetate as the sole end product via Ac-CoA with redox balance, requiring no redox reactions from Ac-CoA through acetate. Therefore, it is beneficial to use the WLP to produce acetone with regard to the redox balance as well.

In this report, we successfully engineered a thermophilic acetogen $M$. thermoacetica for autotrophic acetone production from syngas. Acetone productivity improves by partial deletion of the production pathway for acetate used as a substrate as well as for energy conservation. $M$. thermoacetica grows at a temperature higher than the boiling point of acetone $\left(58^{\circ} \mathrm{C}\right)$; therefore, thermophilic processes of gas fermentation producing volatile chemicals could be built and evaluated. Although further study would be needed to improve the productivity for realization of the industrial applications, the gas fermentation process can be simpler and more cost-effective than before by incorporating a purification process by distillation of the acetone produced from gaseous substrates.

\section{Supplementary Information}

The online version contains supplementary material available at https://doi. org/10.1186/s13568-021-01220-w.

Additional file 1: Figure S1. Schematic representation of energy conservation in acetone-producing Moorella thermoacetica.

\section{Acknowledgements}

The authors would like to thank Enago (www.enago.jp) for the English language review.

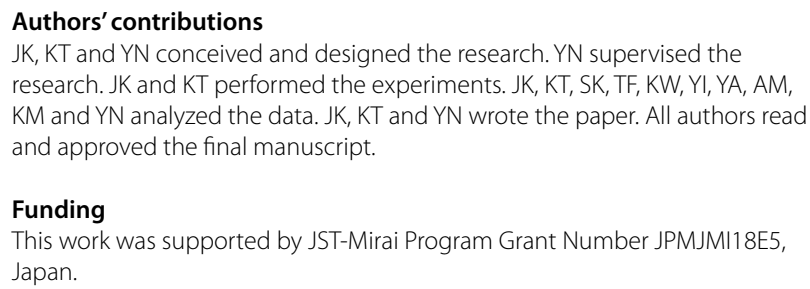

Funding

This work was supported by JST-Mirai Program Grant Number JPMJMI18E5, Japan.

Availability of data and materials

All data collected or analyzed during this study are included in this published article.

\section{Declarations}

Ethics approval and consent to participate

Not applicable.

\section{Consent for publication}

Not applicable.

\section{Competing interests}

This work has been included in a patent application by Hiroshima University.

\section{Author details}

${ }_{1}^{1}$ Graduate School of Integrated Sciences for Life, Hiroshima University, 1-3-1 Kagamiyama, Higashihiroshima, Hiroshima 739-8530, Japan. ${ }^{2}$ National Institute of Advanced Industrial Science and Technology (AIST), 3-11-32 Kagamiyama, Higashihiroshima, Hiroshima 739-0046, Japan.

Received: 4 April 2021 Accepted: 9 April 2021

Published online: 23 April 2021

\section{References}

Anbarasan P, Baer ZC, Sreekumar S, Gross E, Binder JB, Blanch HW, Clark DS, Toste FD (2012) Integration of chemical catalysis with extractive fermentation to produce fuels. Nature 491(7423):235-239. https://doi.org/10. 1038/nature11594

Banerjee A, Leang C, Ueki T, Nevin KP, Lovley DR (2014) Lactose-inducible system for metabolic engineering of Clostridium ljungdahlii. Appl Environ Microbiol 80(8):2410-2416. https://doi.org/10.1128/AEM.03666-13

Bermejo LL, Welker NE, Papoutsakis ET (1998) Expression of Clostridium acetobutylicum ATCC 824 genes in Escherichia coli for acetone production and acetate detoxification. Appl Environ Microbiol 64(3):1079-1085. https:// doi.org/10.1128/AEM.64.3.1079-1085.1998

Breitkopf R, Uhlig R, Drenckhan T, Fischer RJ (2016) Two propanediol utilization-likeproteins of Moorella thermoacetica with phosphotransacetylase activity. Extremophiles 20(5):653-61. https://doi.org/10.1007/ s00792-016-0854-6

Claassens NJ, Sousa DZ, Dos Santos VA, de Vos WM, van der Oost J (2016) Harnessing the power of microbial autotrophy. Nat Rev Microbiol 14(11):692-706. https://doi.org/10.1038/nrmicro.2016.130

Durre P, Eikmanns BJ (2015) C1-carbon sources for chemical and fuel production by microbial gas fermentation. Curr Opin Biotechnol 35:63-72. https://doi.org/10.1016/j.copbio.2015.03.008

Fast AG, Papoutsakis ET (2012) Stoichiometric and energetic analyses of non-photosynthetic $\mathrm{CO}_{2}$-fixation pathways to support synthetic biology strategies for production of fuels and chemicals. Curr Opin Chem Eng 1(4):380-395. https://doi.org/10.1016/j.coche.2012.07.005

Guzman LM, Belin D, Carson MJ, Beckwith J (1995) Tight regulation, modulation, and high-level expression by vectors containing the arabinose $P_{B A D}$ promoter. J Bacteriol 177(14):4121-4130. https://doi.org/10.1128/jb.177. 14.4121-4130.1995

Hoffmeister S, Gerdom M, Bengelsdorf FR, Linder S, Fluchter S, Ozturk H, Blumke W, May A, Fischer RJ, Bahl H, Durre P (2016) Acetone production with metabolically engineered strains of Acetobacterium woodii. Metab Eng 36:37-47. https://doi.org/10.1016/j.ymben.2016.03.001

Hu P, Chakraborty S, Kumar A, Woolston B, Liu H, Emerson D, Stephanopoulos $G$ (2016) Integrated bioprocess for conversion of gaseous substrates to liquids. Proc Natl Acad Sci USA 113(14):3773-3778. https://doi.org/10. 1073/pnas.1516867113

Hungate RE (1969) Chapter IV a roll tube method for cultivation of strict anaerobes. In: Methods in microbiology. Academic Press, New York, pp $117-132$

Iwasaki Y, Kita A, Yoshida K, Tajima T, Yano S, Shou T, Saito M, Kato J, Murakami K, Nakashimada Y (2017) Homolactic acid fermentation by the genetically engineered thermophilic homoacetogen Moorella thermoacetica ATCC 
39073. Appl Environ Microbiol 83(8):1-13. https://doi.org/10.1128/AEM. 00247-17

Jiang Y, Xu C, Dong F, Yang Y, Jiang W, Yang S (2009) Disruption of the acetoacetate decarboxylase gene in solvent-producing Clostridium acetobutylicum increases the butanol ratio. Metab Eng 11(4-5):284-291. https://doi. org/10.1016/j.ymben.2009.06.002

Jones SW, Fast AG, Carlson ED, Wiedel CA, Au J, Antoniewicz MR, Papoutsakis ET, Tracy BP (2016) $\mathrm{CO}_{2}$ fixation by anaerobic non-photosynthetic mixotrophy for improved carbon conversion. Nat Commun 7:12800. https:// doi.org/10.1038/ncomms12800

Kerby R, Zeikus JG (1983) Growth of Clostridium thermoaceticum on $\mathrm{H}_{2} / \mathrm{CO}_{2}$ or CO as energy-source. Curr Microbiol 8(1):27-30. https://doi.org/10.1007/ Bf01567310

Kita A, Iwasaki Y, Sakai S, Okuto S, Takaoka K, Suzuki T, Yano S, Sawayama S, Tajima T, Kato J, Nishio N, Murakami K, Nakashimada Y (2013) Development of genetic transformation and heterologous expression system in carboxydotrophic thermophilic acetogen Moorella thermoacetica. J Biosci Bioeng 115(4):347-352. https://doi.org/10.1016/j.jbiosc.2012.10.013

Kopke M, Gerth ML, Maddock DJ, Mueller AP, Liew F, Simpson SD, Patrick WM (2014) Reconstruction of an acetogenic 2,3-butanediol pathway involving a novel NADPH-dependent primary-secondary alcohol dehydrogenase. Appl Environ Microbiol 80(11):3394-3403. https://doi.org/10.1128/ AEM.00301-14

Liew F, Martin ME, Tappel RC, Heijstra BD, Mihalcea C, Kopke M (2016) Gas fermentation-a flexible platform for commercial scale production of lowcarbon-fuels and chemicals from waste and renewable feedstocks. Front Microbiol 7:694. https://doi.org/10.3389/fmicb.2016.00694

Loder AJ, Zeldes BM, Garrison GD 2nd, Lipscomb GL, Adams MW, Kelly RM (2015) Alcohol selectivity in a synthetic thermophilic n-butanol pathway is driven by biocatalytic and thermostability characteristics of constituent enzymes. Appl Environ Microbiol 81(20):7187-7200. https://doi.org/10. 1128/AEM.02028-15

Luo H, Ge L, Zhang J, Ding J, Chen R, Shi Z (2016) Enhancing acetone biosynthesis and acetone-butanol-ethanol fermentation performance by co-culturing Clostridium acetobutylicum/Saccharomyces cerevisiae integrated with exogenous acetate addition. Bioresour Technol 200:111-120. https://doi.org/10.1016/j.biortech.2015.09.116

Miura T, Kita A, Okamura Y, Aki T, Matsumura Y, Tajima T, Kato J, Nakashimada Y (2014) Evaluation of marine sediments as microbial sources for methane production from brown algae under high salinity. Bioresour Technol 169:362-366. https://doi.org/10.1016/j.biortech.2014.07.013

Peters JW, Schut GJ, Boyd ES, Mulder DW, Shepard EM, Broderick JB, King PW (2015) Adams MW (2015) [FeFe]- and [NiFe]-hydrogenase diversity, mechanism, and maturation. Biochim Biophys Acta 6:1350-1369. https:// doi.org/10.1016/j.bbamcr.2014.11.021

Pierce E, Xie G, Barabote RD, Saunders E, Han CS, Detter JC, Richardson P, Brettin TS, Das A, Ljungdahl LG, Ragsdale SW (2008) The complete genome sequence of Moorella thermoacetica (f. Clostridium thermoaceticum). Environ Microbiol 10(10):2550-73. https://doi.org/10.1111/j.1462-2920. 2008.01679.x

Redl S, Sukumara S, Ploeger T, Wu L, Olshoj Jensen T, Nielsen AT, Noorman $\mathrm{H}$ (2017) Thermodynamics and economic feasibility of acetone production from syngas using the thermophilic production host Moorella thermoacetica. Biotechnol Biofuels 10(1):150. https://doi.org/10.1186/ s13068-017-0827-8

Schafer A, Tauch A, Jager W, Kalinowski J, Thierbach G, Puhler A (1994) Small mobilizable multi-purpose cloning vectors derived from the Escherichia coli plasmids pK18 and pK19: selection of defined deletions in the chromosome of Corynebacterium glutamicum. Gene 145(1):69-73. https://doi. org/10.1016/0378-1119(94)90324-7

Schuchmann K, Muller V (2014) Autotrophy at the thermodynamic limit of life: a model for energy conservation in acetogenic bacteria. Nat Rev Microbiol 12(12):809-821. https://doi.org/10.1038/nrmicro3365

Shaw AJ, Miller BB, Rogers SR, Kenealy WR, Meola A, Bhandiwad A, Sillers WR, Shikhare I, Hogsett DA, Herring CD (2015) Anaerobic detoxification of acetic acid in a thermophilic ethanologen. Biotechnol Biofuels 8(1):75. https://doi.org/10.1186/s13068-015-0257-4

Sonnleitner B, Fiechter A (1983) Advantages of using thermophiles in biotechnological processes: expectations and reality. Trends Biotechnol 1(3):74-80. https://doi.org/10.1016/0167-7799(83)90056-2

Straub CT, Bing RG, Otten JK, Keller LM, Zeldes BM, Adams MWW, Kelly RM (2020) Metabolically engineered Caldicellulosiruptor bescii as a platform for producing acetone and hydrogen from lignocellulose. Biotechnol Bioeng 117(12):3799-3808. https://doi.org/10.1002/bit.27529

Tanner RS (1989) Monitoring sulfate-reducing bacteria - comparison of enumeration media. J Microbiol Methods 10(2):83-90. https://doi.org/10 1016/0167-7012(89)90004-3

Tanner RS, Miller LM, Yang D (1993) Clostridium ljungdahlii sp nov, an acetogenic species in clostridial rRNA homology group I. Int J Syst Bacteriol 43(2):232-6. https://doi.org/10.1099/00207713-43-2-232

Wiesenborn DP, Rudolph FB, Papoutsakis ET (1989) Coenzyme A transferase from Clostridium acetobutylicum ATCC 824 and its role in the uptake of acids. Appl Environ Microbiol 55(2):323-329. https://doi.org/10.1128/ AEM.55.2.323-329.1989

Xu M, Zhao J, Yu L, Tang IC, Xue C, Yang ST (2015) Engineering Clostridium acetobutylicum with a histidine kinase knockout for enhanced n-butanol tolerance and production. Appl Microbiol Biotechnol 99(2):1011-1022. https://doi.org/10.1007/s00253-014-6249-7

Zeldes BM, Straub CT, Otten JK, Adams MWW, Kelly RM (2018) A synthetic enzymatic pathway for extremely thermophilic acetone production based on the unexpectedly thermostable acetoacetate decarboxylase from Clostridium acetobutylicum. Biotechnol Bioeng 115(12):2951-2961. https://doi.org/10.1002/bit.26829

Zhou J, Zhang H, Zhang Y, Li Y, Ma Y (2012) Designing and creating a modularized synthetic pathway in cyanobacterium Synechocystis enables production of acetone from carbon dioxide. Metab Eng 14(4):394-400. https:// doi.org/10.1016/j.ymben.2012.03.005

\section{Publisher's Note}

Springer Nature remains neutral with regard to jurisdictional claims in published maps and institutional affiliations.

\section{Submit your manuscript to a SpringerOpen ${ }^{\circ}$ journal and benefit from:}

- Convenient online submission

- Rigorous peer review

- Open access: articles freely available online

- High visibility within the field

- Retaining the copyright to your article

Submit your next manuscript at $\boldsymbol{\nabla}$ springeropen.com 